\title{
Search for the decay of the Higgs boson into two nMSSM pseudo-scalar particles
}

\author{
Dr. Michel Janus ${ }^{* \dagger}$ \\ II. Physikalisches Institut, Georg-August Universität Göttingen \\ E-mail: janusecern.ch
}

The next-to-minimal supersymmetric Standard Model (nMSSM) predicts the existence of a light pseudo-scalar boson " $a$ " and the decay of the Higgs boson into a pair of such particles. This particle is searched for in several final states sensitive to different decay modes and mass ranges of the hypothetical pseudo-scalar, using ATLAS data amounting to either $20.3 \mathrm{fb}^{-1}$ of $p$ - $p$ collisions at $\sqrt{s}=8 \mathrm{TeV}$ or between $3.2 \mathrm{fb}^{-1}$ and $36.1 \mathrm{fb}^{-1}$ at $\sqrt{s}=13 \mathrm{TeV}$.

The European Physical Society Conference on High Energy Physics

5-12 July, 2017

Venice

* Speaker.

${ }^{\dagger}$ On behalf of the ATLAS collaboration. 


\section{Introduction}

The discovery of a Higgs boson in 2012 [1],[2] raises an exciting question. Is it the Higgs boson of the Standard Model (SM) or is it part of an extended Higgs sector? So far, the observed Higgs boson seems consistent with the predictions for the single Higgs Boson included in the SM. But the current precision of measurements leaves room for new physics, e.g. the branching ratio (BR) into non-SM particles is only constrained to be $30 \%$ or less currently [3]. This is mainly due to the decays into light coloured particles being difficult to access in the LHC environment.

Many models for new physics require an extended Higgs sector [4]. One well-motivated example is the next-to-minimal supersymmetric Standard Model (nMSSM), which contains two Higgs doublets and an additional electro-weak scalar. These mix to form two pseudo-scalar particles $\left(a_{1}, a_{2}\right)$ and three scalar ones $\left(h_{1}, h_{2}, h_{3}\right)$. If the lightest pseudo-scalar is significantly lighter than $m_{h}=125$ $\mathrm{GeV}$, the decay $h \rightarrow a a$ would be possible. Due to the different dominant decay modes that would result, three mass regions $m_{a}<2 m_{\tau}, 2 m_{\tau}<m_{a}<2 m_{b}$ and $m_{a}>2 m_{b}$ need to be considered, where $m_{a}$ is the light pseudo-scalar mass and $m_{\tau}$ and $m_{b}$ are the masses of the $\tau$-lepton and the $b$-quark, respectively. In these proceedings searches at the ATLAS experiment for this decay in several final states are presented covering the range $3.7<m_{a}<62 \mathrm{GeV}$. The considered final states include $\mu \mu \tau \tau, 4 \gamma, 4 b$ and for the first time the 4 lepton final state. These searches were performed using the ATLAS [6] data of $p$ - $p$ collisions at the LHC amounting to either $20.3 \mathrm{fb}^{-1}$ at $\sqrt{s}=8 \mathrm{TeV}$ (Secs. 2 and 3) or between $3.2 \mathrm{fb}^{-1}$ (Sec. 4) and $36.1 \mathrm{fb}^{-1}$ (Sec. 5) at $\sqrt{s}=13 \mathrm{TeV}$ in the ATLAS data.

\section{The $\mu \mu \tau \tau$ final state}

In this search $m_{h}=125 \mathrm{GeV}$ is assumed while the mass of the pseudo-scalar just needs to be above the $\tau$-pair threshold $m_{a}=3.7-50 \mathrm{GeV}$. Subsequently one pseudo-scalar decays to $\tau$-leptons and the second one to muons.

The signature searched for includes one leptonic $\tau$ decay and the second $\tau$ is identified by one or three high $p_{T}$ tracks [5]. The most sensitive search range is $m_{a}<10 \mathrm{GeV}$. The decay to muons, although it has a much lower BR, allows for easy trigger selection and extraction of the signal as a narrow peak in the dimuon invariant mass spectrum $m_{\mu \mu}$. Dominant backgrounds comprise $t \bar{t}$, Drell-Yan, diboson production and low mass resonances $(J / \psi, Y)$. The non-resonant background is estimated by fitting the mass sidebands with a 4th-order polynomial, while the signal and resonant backgrounds are fitted using double-sided crystal ball functions. Figure 1 shows one of the signal regions, where one $\tau$ decays to an electron. No excess over the SM background is observed here nor in the signal region with a muon. Therefore limits as function of $m_{a}$ are set as shown in Fig. 1, under the assumption that the pseudo-scalar does not decay into quarks.

\section{The $4 \gamma$ final state}

An inclusive search in a final state with at least three photons can also be interpreted in the context of the nMSSM [7]. The photon $p_{T}$ thresholds are kept as low as possible to allow sensitivity 

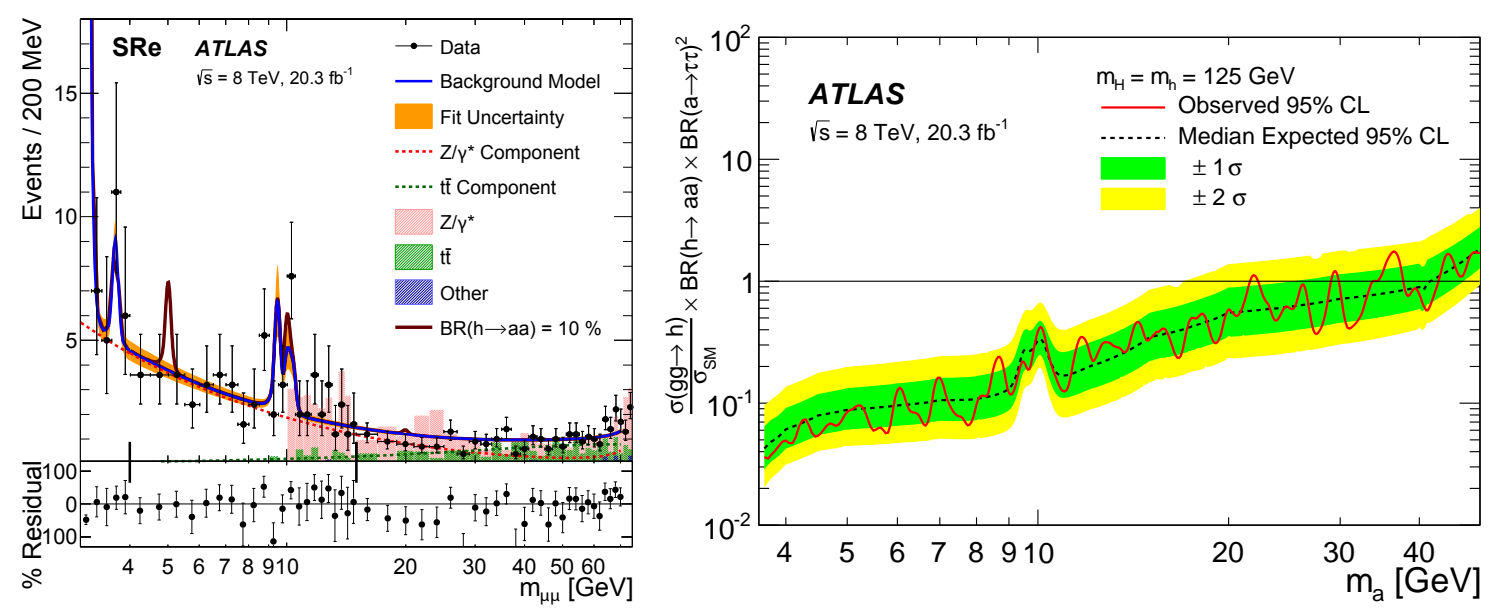

Figure 1: Distribution of the di-muon invariant mass in the signal region where one $\tau$-lepton decays to an electron (left) and limit on production cross section and decay branching ratio as a function of $m_{a}$ (right) [5].

to a mass range of $10<m_{a}<62 \mathrm{GeV}$, while at the same time requiring very restrictive identification and isolation criteria to reduce backgrounds from electrons or jets reconstructed as photons. This background is modelled using data-driven methods to avoid large uncertainties from simulating the misidentification rate of the photon identification. Both signal models where the pair of pseudoscalars result from the decay of the discovered Higgs particle as well as from a hypothetical heavier Higgs boson are considered. Depending on the signal model, the signal extraction is performed by a fit to the mass of the di-photon pairing that yields the smallest combinatorial error. The mass of the second and third leading $p_{T}$ photon, which is used for the signal model with $m_{h}=125 \mathrm{GeV}$, is shown in Fig. 2 (left).

As can be seen, no excess of data above the SM background is observed and the resulting limits on the cross section times branching ratio versus $m_{a}$ are shown in Fig. 2 (right).

\section{The $4 b$ final state}

To reach sensitivity also to low pseudo-scalar masses $\left(m_{a}=20-60 \mathrm{GeV}\right)$ in the decay to four $b$-quarks, the associated production of a Higgs boson with a $W$ boson, where the $W$ decays leptonically, is considered [8]. The lepton from the $W$ decay allows for efficient trigger selection, while avoiding harsh $p_{T}$ requirements on the b-jets. To maximize acceptance, events with at least three jets, two of which need to be tagged as b-jets, are selected. The dominant SM background consists of $t \bar{t}$ production in association with further heavy flavour quarks. Other backgrounds estimated from simulation include production of gauge bosons and single top quarks. The contribution from other multi-jet processes are estimated from data using the so-called matrix method applied to the lepton isolation criterion. Depending on the jet and b-jet multiplicity, events are categorized into signal and control regions. For the signal extraction, a boosted decision tree (BDT) is used, which 

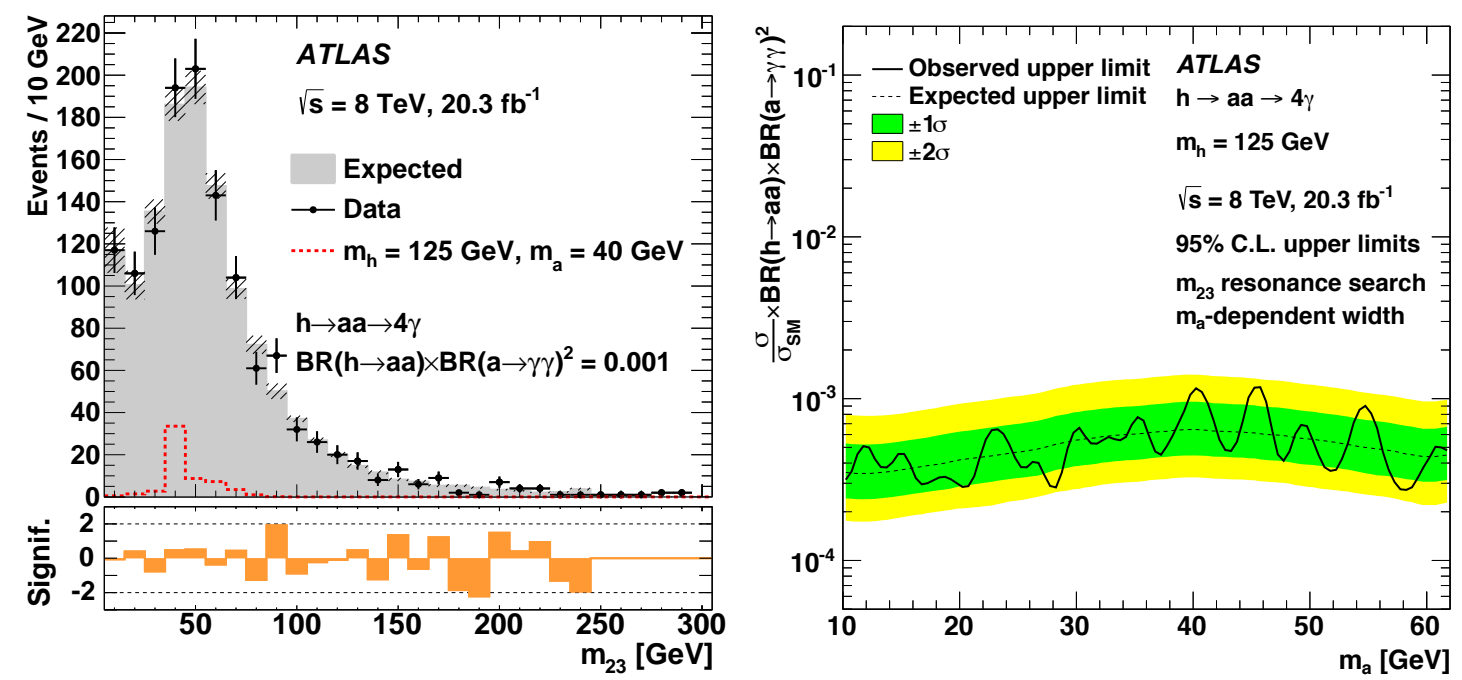

Figure 2: Distribution of the invariant mass of the second- and third-leading photons $m_{23}$ in the $4 \gamma$ final state (left) and limit on production cross section and decay branching ratio as a function of $m_{a}$ (right) [7].

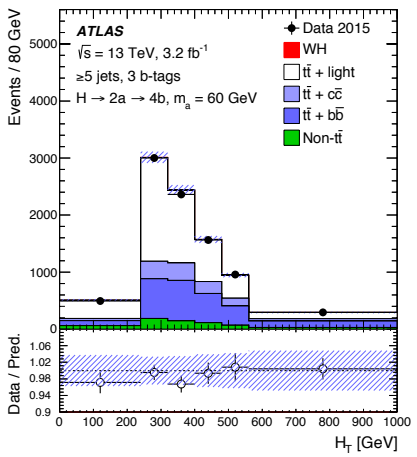

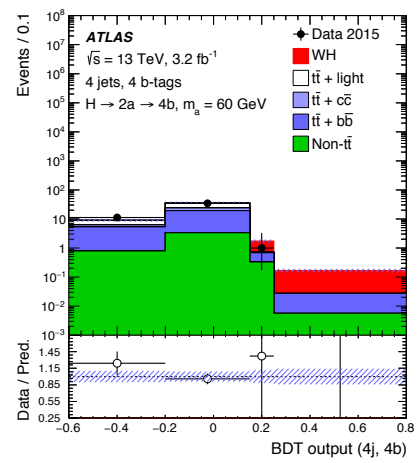

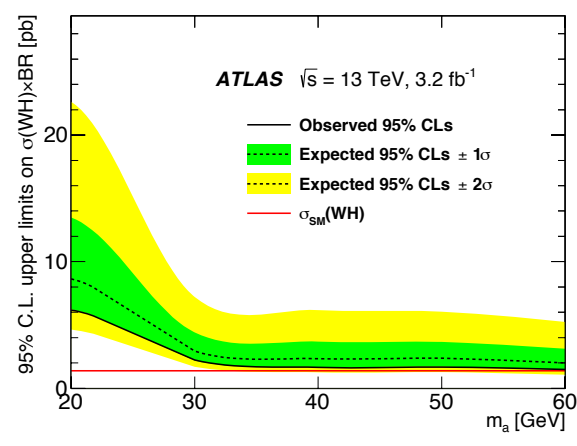

Figure 3: The scalar sum of jet momenta $H_{T}$ in one of fitted control regions (left) and BDT distribution in one signal region (right) as well as the resulting limits on the production cross section and decay branching ratio as a function of $m_{a}$ (middle) [8]. 


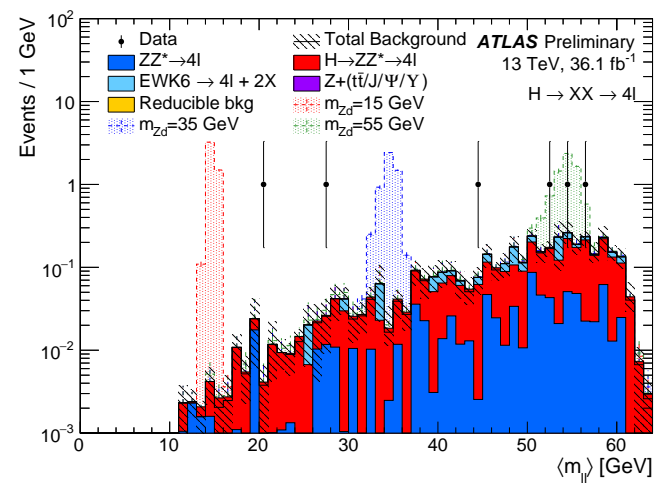

\section{The 4 lepton final state}

For the first time ATLAS presented the results from a search for the decay $h \rightarrow a a \rightarrow 4$ leptons [9]. In this search the experimental signature consists of two opposite-sign, same flavour, light lepton pairs. In the case where all four leptons are of the same type, the pairing that results in the smallest mass difference between pairs is chosen and pairs are ordered by closeness to the $Z$ boson mass. Two sets of selection criteria are optimized separately for low (1-15 GeV) and high (15$60 \mathrm{GeV}$ ) pseudo-scalar masses. The low mass selection only considers the four muon final state. Also, the four-lepton mass is required to be consistent with the observed Higgs mass. Vetoes on di-lepton mass are used to reject backgrounds from quarkonia. In the high mass selection, a $Z$-mass veto and angular separation between leptons is required in addition. The dominant backgrounds that contribute to both signal regions are di- $Z$ production from SM Higgs decays and from the continuum. Leptonic heavy flavour decays become important in the low mass selection. Signs of potential signal are extracted by a profile maximum likelihood fit to the average di-lepton pair mass distribution $\left\langle m_{l l}>\right.$ as shown in Fig. 4 (left). Although one event is observed at $\left\langle m_{l l}>\approx 20 \mathrm{GeV}\right.$ over the very small SM background, the significance of this is below $3 \sigma$ once the look-elsewhere effect is taken into account. Since no clear sign of a signal is observed, limits on $B R(h \rightarrow a a)$ are set accordingly, which are shown in Fig. 4 (right). The additional interpretation under the signal hypothesis of the Higgs decaying to dark $Z$ bosons is also shown.

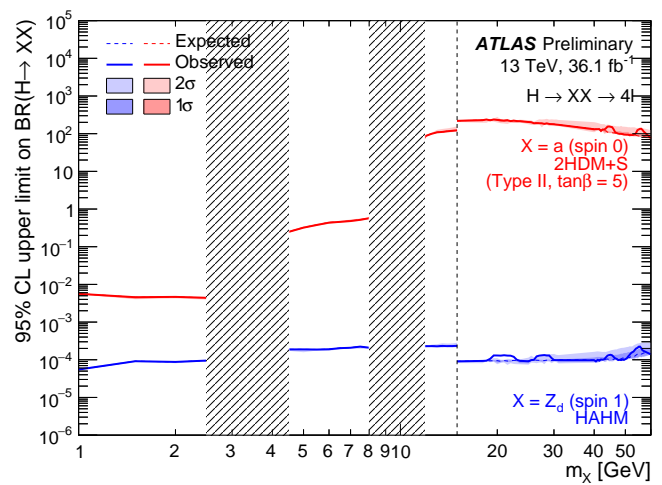

Figure 4: Distribution of the average invariant di-lepton mass in the low mass signal region (right) and limit on the decay branching ratio $h \rightarrow a a / Z_{d} Z_{d}$ as a function of $m_{a}$ (right) [9].

\section{Summary}

Four different searches, including the $\mu \mu \tau \tau, 4 \gamma, 4 b$ and 4 lepton final states, for the decay of the newly discovered Higgs boson to a pair of light pseudo-scalars $(a)$ in a mass range $3.7<$ $m_{a}<62 \mathrm{GeV}$ were presented. The dominant decay modes vary according to the mass of the pseudo-scalar, depending on whether it is over the di- $\tau$ - or di-b-mass threshold. No excess over the background expectations in the different search modes is observed and therefore the branching ratio for the decays of the Higgs boson to light pseudo-scalars as a function of pseudo-scalar mass 
are further constrained. With the existing datasets, these limits however do not completely exclude many well-motivated extensions to the SM Higgs sector and this topic will remain relevant for the duration of the LHC and high luminosity LHC projects.

\section{References}

[1] ATLAS Collaboration, Phys.Lett.B716,1(2012)[arXiv:1207.7214[hep-ex]].

[2] CMS Collaboration, Phys.Lett.B716,30(2012)[arXiv:1207.7235[hep-ex]].

[3] ATLAS and CMS Collaborations, JHEP1608,045(2016)[arXiv:1606.02266[hep-ex]].

[4] D.Curtin et al., PhysRevD.90, 075004 (2014)[arXiv:1312.4992 [hep-ph]].

[5] ATLAS Collaboration, Phys.Rev.D92,no.5,052002(2015)[arXiv:1505.01609[hep-ex]].

[6] ATLASCollaboration, JINST3,S08003(2008).

[7] ATLASCollaboration, Eur.Phys.J.C76,no.4,210(2016)[arXiv:1509.05051[hep-ex]].

[8] ATLAS Collaboration, Eur. Phys. J. C 76 (2016) 605[arXiv:1606.08391[hep-ex]].

[9] ATLAS Collaboration, ATLAS-CONF-2017-042, CERN 2017. 